\title{
Performance Comparison of Quantitative Methods for PMU Data Event Detection with Noisy Data
}

\author{
L. Souto, S. Herraiz, J. Meléndez \\ Intelligent Systems \& Control Engineering group \\ Universitat de Girona \\ Girona, Girona, Spain \\ laiz.souto, sergio.herraiz, joaquim.melendez AT udg.edu
}

\begin{abstract}
This article compares distinct signal-based and knowledge-based approaches often applied to process and detect events in vast amounts of data collected by phasor measurement units (PMU). The computation times and the accuracy of correct event detections are tested and evaluated in a 1-hour data file from the UT-Austin Independent Texas Synchrophasor Network with phasor quantities plus an additive noise gathered at different PMU substations. A sliding time window is considered to build a representative model of the system operating conditions on the fly and search for power system phenomena as soon as new data are available.
\end{abstract}

Index Terms-fault detection, phasor measurement units, power system faults, principal component analysis

\section{INTRODUCTION}

$\mathbf{E}$ LECTRIC power systems are becoming increasingly complex, albeit decreasingly tolerant to faults and power quality concerns. As a result, any types of abnormal operating conditions must be detected and diagnosed as early as possible to minimize performance degradation and prevent dangerous situations.

Although smart grid infrastructures have the ability to collect massive amounts of data that can be exploited to identify anomalies at different locations and voltage levels, it is a difficult task to visualize and identify patterns, outliers, and abnormal behaviors at relevant scales. In this context, an automated methodology capable of processing huge amounts of data and detecting anomalies of different nature may contribute to the task.

Overall, the techniques applied to fault detection and diagnosis in power systems (in this article, fault refers to distinct anomalies in power system operation) can be classified as model-based, signal-based, knowledge-based, and hybrid approaches [1], [2]. Model-based fault detection techniques request a model of the system or process for evaluation of the consistency between the measured outputs and the model predicted outputs. They can be categorized as deterministic (e.g. solvable with linear matrix inequalities) and

This research was supported by the European Union's Horizon 2020 research and innovation programme, call LCE-01-2016-2017, under the auspices of the project "Renewable penetration levered by Efficient Low Voltage Distribution grids", grant agreement number 773715, and University of Girona scholarship.

/20/\$31.00 @ 2020 IEEE stochastic (e.g. Kalman filters). Signal-based fault detection techniques rely on measured signals for feature extraction in either or both time domain and frequency domain. Timedomain features include continuous monitoring of average, standard deviation, phases, slope, peak, and root mean square magnitudes (e.g. min-max difference, differential function), whereas frequency-domain features include spectrum analysis (e.g. discrete Fourier transform, Yule-Walker spectral method) [3]. Knowledge-based fault detection methods rely on the availability of large amounts of historical data for extraction of the underlying knowledge of a given process and can be categorized as quantitative or qualitative. Qualitative methods (e.g. expert systems, qualitative trend analysis) are based on a set of rules, whereas quantitative methods rely on pattern recognition and can be statistical (e.g. principal component analysis, independent component analysis, partial least squares, support vector machines) or non-statistical (e.g. neural networks, fuzzy logic). Finally, hybrid approaches for fault detection consist of a combination of the fault detection strategies previously described.

The usage of signal-based and statistical quantitative knowledge-based fault detection methods is advised when the monitored process is non-stationary and the evaluation has to adapt to changing operating conditions, since model-based fault detection techniques require an accurate input-output description which is not always available, whereas qualitative and non-statistical quantitative knowledge-based methods are process specific and are computationally more expensive than statistical quantitative methods to be re-trained. Nonetheless, there is a lack of consensus about the most effective approach to detect anomalies in power system operation, especially in the presence of noisy data, which adds uncertainties to the analysis.

Fitting into this context, this article provides a comparative analysis of different fault detection approaches with respect to the accuracy of correct event detections in the presence of noisy data. The methods are tested in a 1-hour data file from Texas Synchrophasor Network and applicable to power transmission and distribution networks, relying on electrical quantities gathered at different locations. The operating conditions of the grid are modelled over a sliding window basis, which improves the situational awareness of the analysis.

The text is organized as follows. The signal-based and 
knowledge-based fault detection techniques are presented in Sections II and III, respectively, together with the criteria commonly used to detect data anomalies. Afterwards, Section IV presents a case study, whose results are discussed in Section V. Finally, conclusions are presented in Section VI.

\section{SignAl-BASED FAULT DETECTION METHODS}

\section{A. Fast Fourier transform}

The Fast Fourier transform (FFT) computes the discrete Fourier transform of a signal to quantify its frequency content. For an in-depth explanation, refer to [4].

\section{B. Yule-Walker Spectral method}

The Yule-Walker Spectral method computes the power spectral density of a signal using the autoregressive YuleWalker method. For a thorough explanation, see [5].

\section{Min-Max difference}

This method computes the difference between maximum and minimum values of the signal within the time window. For more details, see [3].

\section{Difference and approximate derivative}

This method computes the maximum difference between consecutive samples of the signal within the time window. The calculation is analogous to the rate of change of frequency (ROCOF). For more details, refer to [3].

\section{KNOWLEDGE-BASED FAULT DETECTION METHODS}

\section{A. Principal Component Analysis}

This method builds a statistical model of the data with dimensionality reduction and detects anomalies by projecting the subsequent data onto the projection subspace. For an indepth explanation, see [6]. In this article, the minimization of the variance reconstruction error (VRE) is considered to select the number of principal components to build the PCA model. For an in-depth explanation, see [7].

\section{B. Support Vector Machines}

This method finds the best hyperplane that separates all data points representing normal operation from those representing abnormal operation and detects anomalies with use of this classification hyperplane. If the data do not allow for a separating hyperplane, Kernel functions and soft margins can be used to separate as many data points as possible. For a thorough explanation, refer to [8].

\section{CASE STUDY}

The event detection techniques are tested with PMU data from the UT-Austin Independent Texas Synchrophasor Network available in [3] (see website). The PMU substations are located at distinct transmission and distribution voltage levels within the Electric Reliability Council of Texas (ERCOT) and provide a good overview of the system [9].

The events that can be found in the data set, illustrated in Fig. 1 to 20, consist of low-frequency voltage oscillations which last no more than a few hundred milliseconds (below

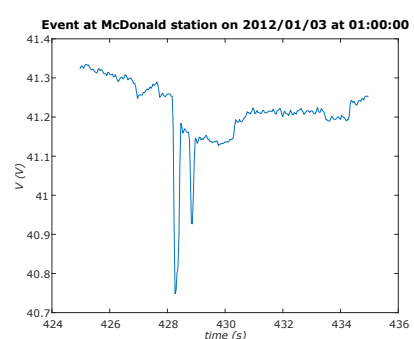

Fig. 1. Event detected between $425 \mathrm{~s}$ and $435 \mathrm{~s}$

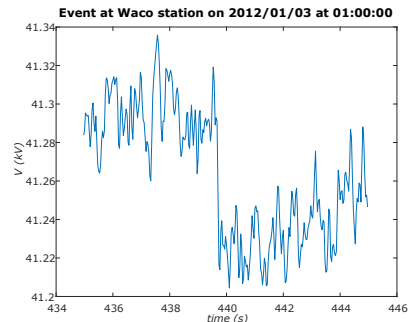

Fig. 2. Event detected between $435 \mathrm{~s}$ and $445 \mathrm{~s}$

$15 \mathrm{~Hz}$, as the PMU sampling rate is $30 \mathrm{~Hz}$ ). All of them can be identified by at least one of the signal-based fault detection methods described in Section II without noise in the data, as shown in Table I. In particular, Fig. 1, 3, 4, 5, 6, 7, 8, 10, 11, 13, 14, 15, 16, 17, 19, and 20 contain impulses, whereas Fig. 2, 9, 12, and 18 contain step changes; the events displayed in Fig. 1, 3, 4, 5, 6, 7, 8, 9, 10, 11, 13, 15, 16, 17, 19, and 20 occurred at the McDonald station (extra low voltage), whereas those displayed in Fig. 2, 12, 14, and 18 occurred at the Waco station (high voltage).

In this article, the signal-based fault detection methods detect anomalies with use of the $3 \sigma$ rule, in accordance to the analysis performed in [3]. Meanwhile, the PCA algorithm relies on square prediction error (SPE) statistics with the $3 \sigma$ rule to detect anomalies, in agreement with the signal-based methodologies aforementioned. Although the limits calculated with the $3 \sigma$ rule are more conservative than those calculated with the analytical method for the SPE statistics, the results are not affected negatively, as shown in Section V. In turn, the SVM classification considers that normal operation is represented by the average values of the data over a sliding time window and that a deviation of $2 \%$ from the average represents abnormal operation, based on the magnitude of the events recorded in Fig. 1 to 20.

The event detection techniques are run over a 10-second sliding window basis, which captures the dynamic timevarying nature of the system and enables adaptation to the most recent operating conditions, and further tested and compared in terms of performance of correct event detections with an additive disturbance represented by a white Gaussian noise $\delta$ with $\operatorname{var}(\delta)=0.25 V^{2}$ added to the original PMU data. This choice results in a signal to noise (SNR) ratio of $40 \mathrm{~dB}$ at the McDonald station, slightly below the $45 \mathrm{~dB}$ approximation suggested in [10]. 


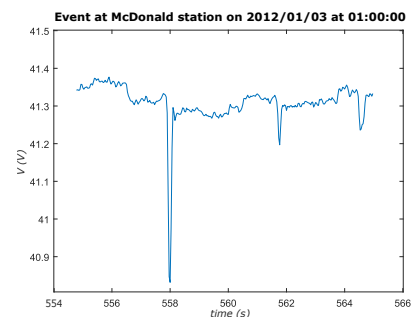

Fig. 3. Event detected between $555 \mathrm{~s}$ and $565 \mathrm{~s}$

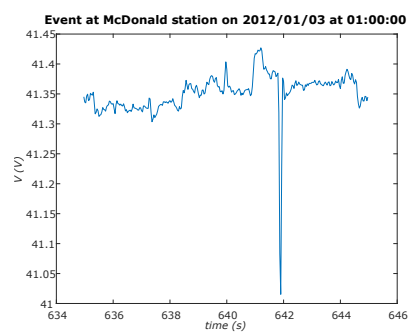

Fig. 4. Event detected between $635 \mathrm{~s}$ and $645 \mathrm{~s}$

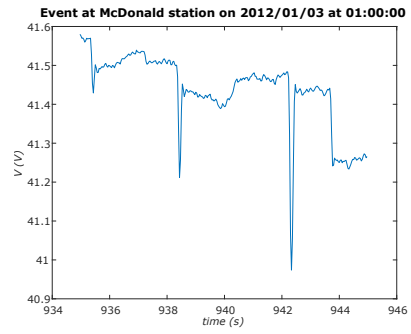

Fig. 5. Event detected between $935 \mathrm{~s}$ and $945 \mathrm{~s}$

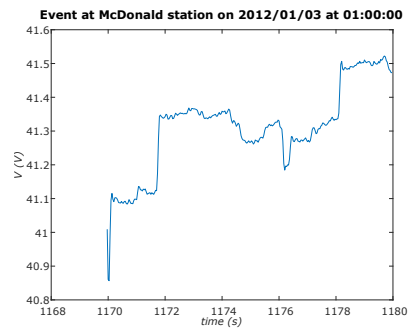

Fig. 6. Event detected between $1170 \mathrm{~s}$ and $1180 \mathrm{~s}$

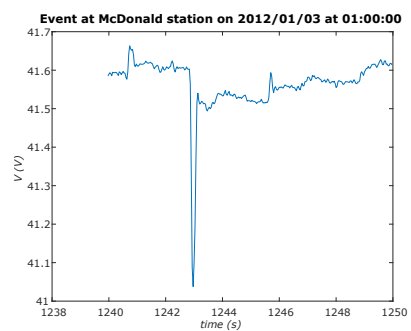

Fig. 7. Event detected between $1240 \mathrm{~s}$ and $1250 \mathrm{~s}$

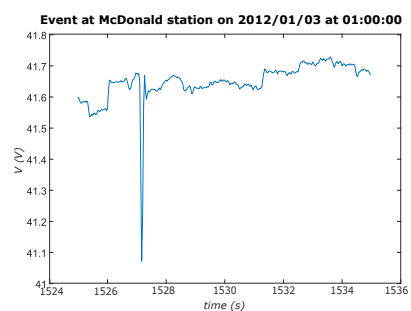

Fig. 8. Event detected between $1520 \mathrm{~s}$ and $1530 \mathrm{~s}$

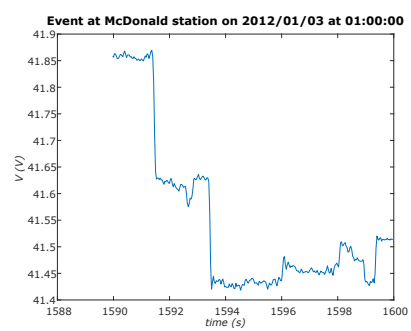

Fig. 9. Event detected between $1590 \mathrm{~s}$ and $1600 \mathrm{~s}$

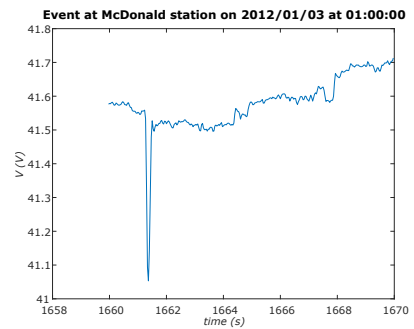

Fig. 10. Event detected between $1660 \mathrm{~s}$ and $1670 \mathrm{~s}$

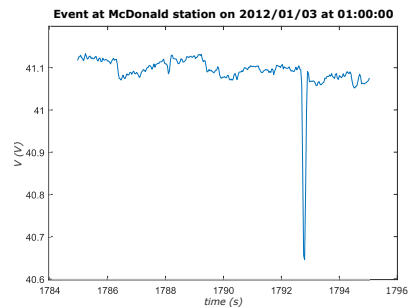

Fig. 11. Event detected between $1785 \mathrm{~s}$ and $1795 \mathrm{~s}$

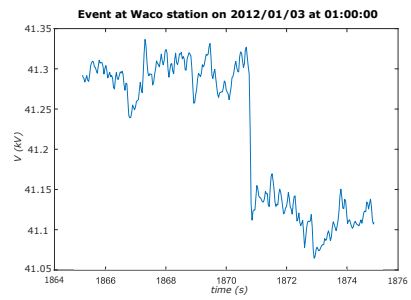

Fig. 12. Event detected between $1865 \mathrm{~s}$ and $1875 \mathrm{~s}$

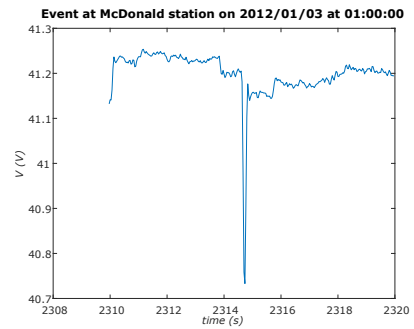

Fig. 13. Event detected between $2310 \mathrm{~s}$ and $2320 \mathrm{~s}$ 


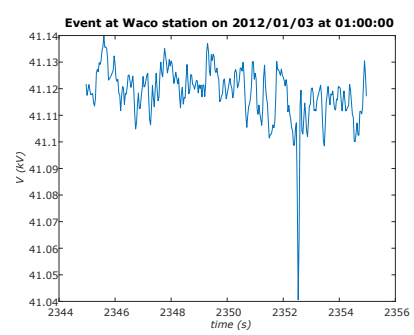

Fig. 14. Event detected between $2345 \mathrm{~s}$ and $2355 \mathrm{~s}$

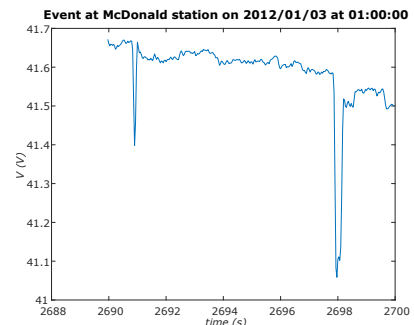

Fig. 15. Event detected between $2690 \mathrm{~s}$ and $2700 \mathrm{~s}$

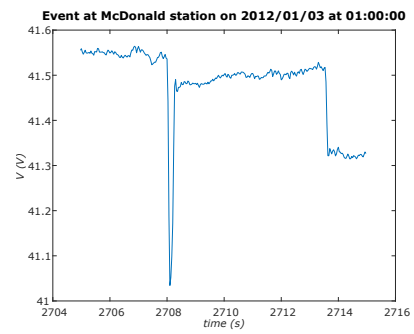

Fig. 16. Event detected between $2705 \mathrm{~s}$ and $2715 \mathrm{~s}$

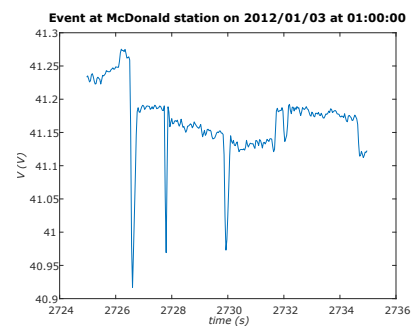

Fig. 17. Event detected between $2725 \mathrm{~s}$ and $2735 \mathrm{~s}$

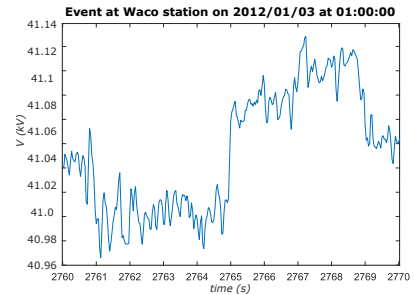

Fig. 18. Event detected between $2760 \mathrm{~s}$ and $2770 \mathrm{~s}$

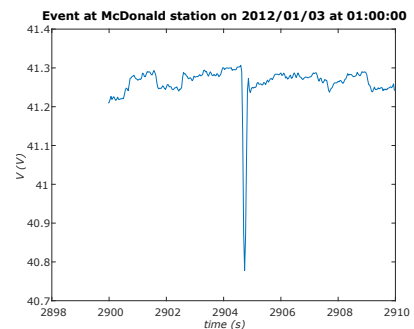

Fig. 19. Event detected between $2900 \mathrm{~s}$ and $2910 \mathrm{~s}$

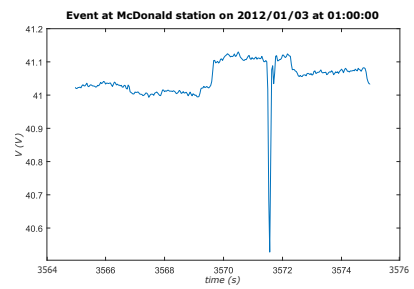

Fig. 20. Event detected between $3565 \mathrm{~s}$ and $3575 \mathrm{~s}$

TABLE I

EVENT DETECTION RESULTS OVER A 10-S WINDOW WITHOUT NOISE

\begin{tabular}{lllllll}
\hline \hline time (s) & FFT & Yule-Walker & Min-Max & Derivative & PCA & SVM \\
\hline 435 & Yes & No & Yes & No & Yes & Yes \\
445 & No & Yes & No & No & Yes & No \\
565 & No & No & Yes & Yes & Yes & Yes \\
645 & No & No & No & Yes & Yes & Yes \\
945 & Yes & No & Yes & No & Yes & Yes \\
1180 & Yes & No & Yes & No & Yes & Yes \\
1250 & Yes & No & Yes & No & Yes & Yes \\
1530 & No & No & Yes & Yes & Yes & Yes \\
1600 & Yes & Yes & No & No & Yes & Yes \\
1670 & No & No & Yes & No & Yes & Yes \\
1795 & No & No & No & Yes & Yes & Yes \\
1875 & No & Yes & Yes & No & Yes & No \\
2320 & No & No & No & Yes & Yes & Yes \\
2355 & No & No & No & Yes & Yes & Yes \\
2700 & Yes & No & Yes & No & Yes & Yes \\
2715 & Yes & No & No & No & Yes & Yes \\
2735 & Yes & No & No & No & Yes & Yes \\
2770 & No & Yes & Yes & No & Yes & No \\
2910 & No & No & No & Yes & Yes & Yes \\
3575 & No & No & Yes & Yes & Yes & Yes \\
\hline \hline
\end{tabular}

\section{RESULTS AND DISCUSSION}

This section presents the results of event detection for the scenarios described in Section IV and compares the accuracy of the methods described. The results of correct event detections in the original data are presented in Table I, whereas the results of correct event detections in the presence of an additive noise are presented in Table II.

Considering the correct detections indicated in Table I, the best performance is obtained with principal component analysis (PCA), which detects all 20 events, followed by support vector machines (SVM), which detects 17 out of 20 events, whereas the results detected with the Fast Fourier transform (FFT), Yule-Walker spectral analysis, min-max difference, and approximate derivative methods are complementary. In particular, the min-max difference detects 11 occurrences, whereas both the FFT and the approximate derivative detect 8 events and the Yule-Walker spectral analysis detects 4 events.

In turn, considering the correct detections indicated in Table II, the best performance is obtained with support vector machines (SVM), which detects 15 out of 20 events, followed by principal component analysis (PCA), which detects 12 out of 20 events. Additionally, it can be noticed in Table II that 18 out of 20 detections are achieved by PCA and SVM combined. Conversely, the Yule-Walker spectral analysis detects 3 events, whereas the min-max difference detects 2 events and both the FFT and the approximate derivative detect 1 event each. 
TABLE II

EVENT DETECTION RESULTS OVER A 10-S WINDOW WITH NOISE

\begin{tabular}{lllllll}
\hline \hline time (s) & FFT & Yule-Walker & Min-Max & Derivative & PCA & SVM \\
\hline 435 & No & No & No & No & No & Yes \\
445 & No & Yes & No & No & Yes & No \\
565 & No & No & No & No & No & Yes \\
645 & No & No & No & No & No & Yes \\
945 & No & No & No & No & Yes & Yes \\
1180 & No & No & No & No & No & Yes \\
1250 & No & No & No & No & Yes & Yes \\
1530 & No & No & No & No & Yes & Yes \\
1600 & Yes & No & No & No & Yes & No \\
1670 & No & No & No & No & Yes & Yes \\
1795 & No & No & No & No & No & Yes \\
1875 & No & Yes & Yes & No & No & No \\
2320 & No & No & No & No & Yes & Yes \\
2355 & No & No & No & Yes & Yes & No \\
2700 & No & No & No & No & Yes & Yes \\
2715 & No & No & No & No & Yes & Yes \\
2735 & No & No & No & No & Yes & Yes \\
2770 & No & Yes & Yes & No & No & No \\
2910 & No & No & No & No & Yes & Yes \\
3575 & No & No & No & No & No & Yes \\
\hline \hline
\end{tabular}

Alternatively, if only voltage impulses are considered (i.e. all sliding windows but those ending at 445, 1600, 1875, 2770 s), all 16 remaining events are detected with both PCA and SVM without noise according to Table I. In this case, the best performance in the presence of noise is obtained with SVM, which detects 15 out of 16 events, followed by PCA, which detects 10 out of 16 events.

Moreover, it is expected that the additive noise will affect most significantly the events detected at low voltage distribution level for its magnitude. This is verified in Tables I and II, as the results obtained for the sliding windows ending at 445 and $1670 \mathrm{~s}$ remain the same for all methods. However, the step changes in the sliding windows ending at 1875 and 2770 $\mathrm{s}$ are missed by the PCA algorithm in the presence of noise and detected by both the Yule-Walker spectral method and the min-max difference algorithm.

\section{CONCLUSION}

This article has considered different signal-based and knowledge-based methods for PMU data event detection and compared them in terms of accuracy of correct event detections with noisy data applied to a 1-hour data file from the Texas Synchrophasor Network. The results obtained with this data set indicate that the statistical knowledge-based fault detection techniques present the best performance individually and are more robust to disturbances than signal-based fault detection techniques. Therefore, the usage of knowledge-based techniques is recommended for accurate PMU data event detection.

\section{REFERENCES}

[1] Z. Gao, C. Cecati, and S. X. Ding, "A survey of fault diagnosis and fault-tolerant techniques_-part i: Fault diagnosis with model-based and signal-based approaches," IEEE Transactions on Industrial Electronics, vol. 62, no. 6, pp. 3757-3767, June 2015 .
[2] — "A survey of fault diagnosis and fault-tolerant techniques_-part ii: Fault diagnosis with knowledge-based and hybrid/active approaches," IEEE Transactions on Industrial Electronics, vol. 62, no. 6, pp. 37683774, June 2015.

[3] A. Allen, M. Singh, E. Muljadi, and S. Santoso, "Pmu data event detection: A user guide for power engineers," 2014, link to accompanying zipped data file: http://www.nrel.gov/docs/fy15osti/61664-1.zip.

[4] C. S. Burrus, Fast Fourier Transforms. OpenStax CNX, 2012.

[5] H. Zhang and P. Duhamel, "On the methods for solving yule-walker equations," IEEE Transactions on Signal Processing, vol. 40, no. 12, pp. 2987-3000, Dec 1992.

[6] E. Russell, L. Chiang, and R. Braatz, Data-driven Methods for Fault Detection and Diagnosis in Chemical Processes, ser. Advances in Industrial Control. Springer London, 2000.

[7] S. J. Qin and R. Dunia, "Determining the number of principal components for best reconstruction," Journal of Process Control, vol. 10, no. 2, pp. $245-250,2000$

[8] I. Steinwart and A. Christmann, Support Vector Machines, ser. Information Science and Statistics. Springer New York, 2014.

[9] W. M. Grady and D. Costello, "Implementation and application of an independent texas synchrophasor network," in 2010 63rd Annual Conference for Protective Relay Engineers, March 2010, pp. 1-12.

[10] M. Brown, M. Biswal, S. Brahma, S. J. Ranade, and H. Cao, "Characterizing and quantifying noise in pmu data," in 2016 IEEE Power and Energy Society General Meeting (PESGM), July 2016, pp. 1-5. 